\title{
Toward a Better Understanding on the Mechanism of Ortholithiation. Tuning of Selectivities in the Metalation of Meta-Anisic Acid by an Appropriate Choice of the Base
}

\section{SUPPORTING INFORMATION}

\author{
Thi-Huu Nguyen, ${ }^{a}$ Nguyet Trang Thanh Chau, ${ }^{a}$ Anne-Sophie Castanet, ${ }^{a}$ \\ Kim Phi Phung Nguyen, ${ }^{b}$ and Jacques Mortier ${ }^{* a}$
}

\footnotetext{
a Université du Maine and CNRS, Unité de chimie organique moléculaire et macromoléculaire (UMR 6011), Faculté des sciences, avenue Olivier Messiaen, 72085 Le Mans Cedex 9 (France)

${ }^{\mathrm{b}}$ Université nationale de Ho-Chi-Minh-Ville, École des sciences naturelles, Laboratoire de chimie organique, 283/2 Nguyen van Cu. arrondissement 5, Ho Chi Minh Ville (Vietnam)
} 


\section{Table of contents}

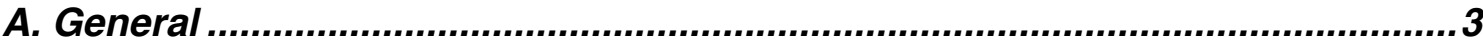

B. 2-Substituted 3-methoxybenzoic acids (10-12, 16a-d, 21 and 23) .................... 3

B1. In situ quench technique. 3-Methoxy-2-(trimethylsilyl)benzoic acid (11) 3

B2. General procedure for the preparation of 2-substitued 3-methoxybenzoic acids

$(10,12,16 a-d, 21$ and 23)

4

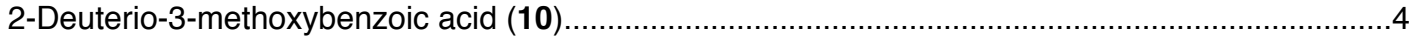

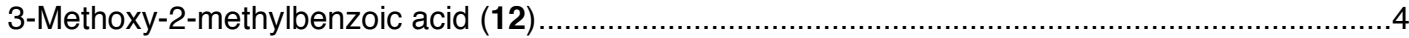

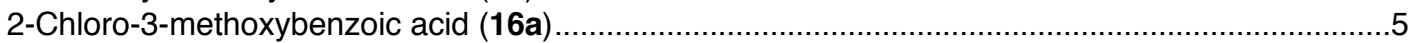

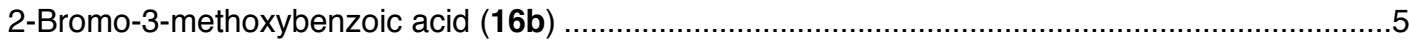

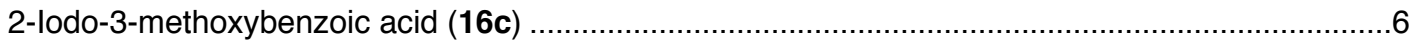

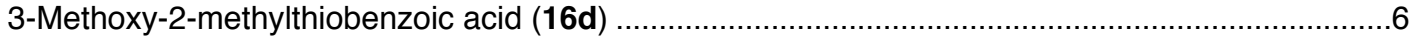

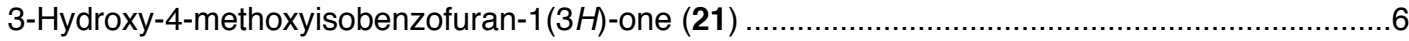

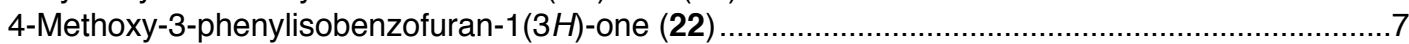

2,6-Di(trimethylsilyl)-3-methoxybenzoic acid (15) (entry 5 of Table 1) ..........................................

C. General procedure for the preparation of 4-substituted 3-methoxybenzoic

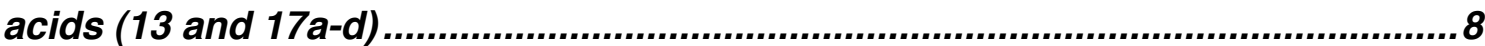

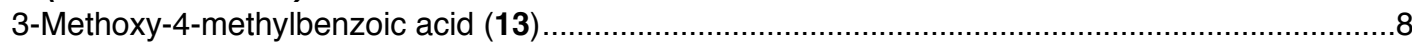

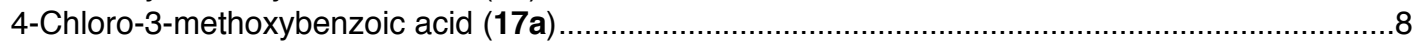

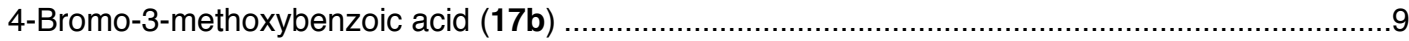

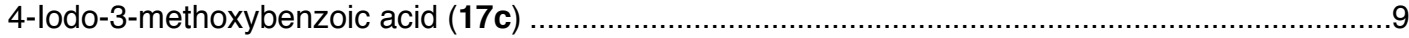

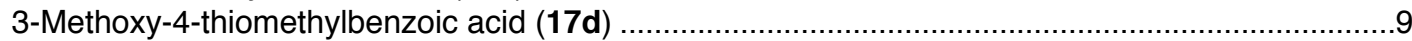

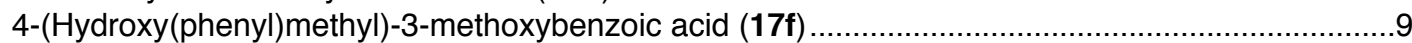

D. General procedure for the preparation of 6-substituted-3-methoxy-2-

(trimethylsilyl)benzoic acids (18, 19a-e and 22) and 6-substitued 3-

methoxybenzoic acids (14 and 20a-d)............................................................ 10

6-Methyl-3-methoxy-2-(trimethylsilyl)benzoic acid (18) .......................................................... 10

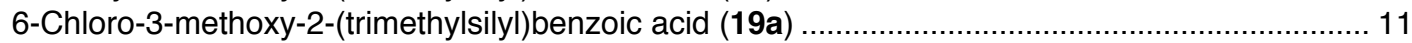

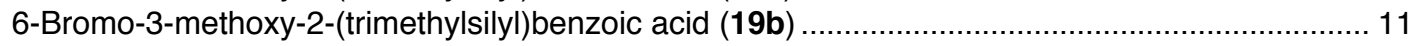

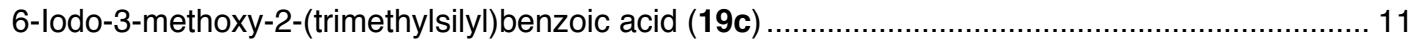

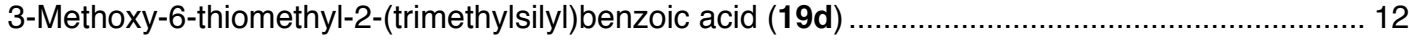

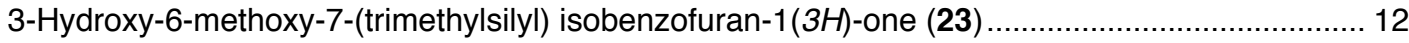

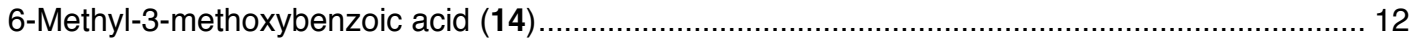

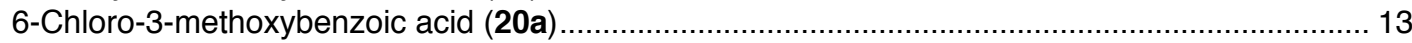

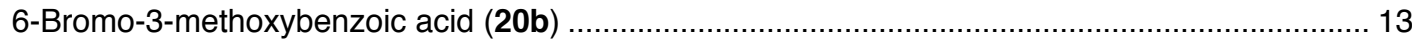

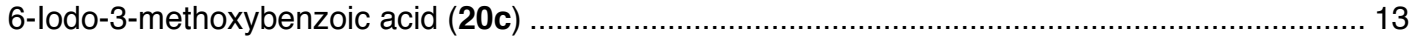

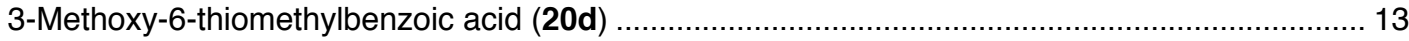




\section{A. General}

For standard working practice, see recent publications (e.g. refs ${ }^{1,2}$ ). Reactions were carried out under argon in oven-dried glassware. Tetrahydrofuran was dried from sodium benzophenone. lodomethane, $\mathrm{N}, \mathrm{N}$-dimethylformamide and benzaldehyde were dried with $\mathrm{CaH}_{2}$ and distilled prior to use. NMR spectra were recorded on a $200-$ or $400-\mathrm{MHz}$ spectrometer. ${ }^{13} \mathrm{C}$ NMR spectra were obtained with broadband proton decoupling. For spectra recorded in $\mathrm{CDCl}_{3}$, chemical shifts were recorded relative to the internal TMS (tetramethylsilane) reference signal. For DMSO$d_{6}$, chemical shifts are given relative to the solvent signals. All melting points are uncorrected.

$n$-BuLi (1.6 M in hexanes) and s-BuLi (1.3 $\mathrm{M}$ in cyclohexane-hexanes) were titrated periodically against 2,5-dimethoxybenzyl alcohol. $N, N, N^{\prime}, N^{\prime}-$ Tetramethyl-1,2ethylenediamine (TMEDA) was distilled from $\mathrm{CaH}_{2}$. Potassium tert-butylate $(t-\mathrm{BuOK})$ was sublimated.

\section{B. 2-Substituted 3-methoxybenzoic acids (10-12, 16a-d, 21 and 23)}

\section{B1. In situ quench technique. 3-Methoxy-2-(trimethylsilyl)benzoic acid (11)}

To a solution of LTMP $(9 \mathrm{mmol})$ in THF $(20 \mathrm{~mL})$ at $-78{ }^{\circ} \mathrm{C}$ were added successively chlorotrimethylsilane $(1.38 \mathrm{~mL}, 10.5 \mathrm{mmol})$ in THF $(5 \mathrm{~mL})$ and 3methoxybenzoic acid (6) $(0.46 \mathrm{~g}, 3 \mathrm{mmol})$ in THF $(5 \mathrm{~mL})$. After being warmed gradually to $0{ }^{\circ} \mathrm{C}$ for $2 \mathrm{~h}$, the soltion was allowed to warm to ambient temperature. Aqueous (2M) $\mathrm{NaOH}$ was added until the $\mathrm{pH}$ reached 10. The aqueous layer was washed with diethyl ether, acidified with aqueous (4M) $\mathrm{HCl}$, and extracted with diethyl ether. The organic layer was dried over $\mathrm{MgSO}_{4}$, filtrated, and concentrated in vacuo.

Schlosser, M. Organometallics in Synthesis. A Manual; Wiley: Chichester, $2^{\text {nd }}$ Edition, 2002.

(a) Cantegril, R.; Mortier, J.; Croisat, D.; Peignier, R. Wo. Pat. 9602138, 1996. (b) Cantegril, R.; Croisat, D.; Desbordes, P.; Guigues, F.; Mortier, J.; Peignier, R.; Vors, J.-P. U. S. Pat. 5945382 1999. 
The residue was purified by colum chromatography on silicagel (cyclohexane/ethyl acetate 90:10, $\left.R_{f}=0.31\right)$ to give $11(75 \%)$ as a white solid (mp 91-92 ${ }^{\circ} \mathrm{C}$ ). ${ }^{1} \mathrm{H}$ NMR $\left(400 \mathrm{MHz}, \mathrm{CDCl}_{3}\right) \rightarrow 0.33(\mathrm{~s}, 9 \mathrm{H}), 3.82(\mathrm{~s}, 3 \mathrm{H}), 7.01(\mathrm{~d}, 1 \mathrm{H}, J=7.8 \mathrm{~Hz}), 7.35-7.64$ $(\mathrm{m}, 2 \mathrm{H}) .{ }^{13} \mathrm{C}$ NMR $\left(50 \mathrm{MHz}, \mathrm{CDCl}_{3}\right) \rightarrow 0.6,54.1,112.1,120.1,126.9,129.0,137.9$, 163.7, 175.1. IR (neat): 2959, 2560, $1673 \mathrm{~cm}^{-1}$. Anal. calcd. for $\mathrm{C}_{11} \mathrm{H}_{16} \mathrm{O}_{3} \mathrm{Si}: \mathrm{C}, 58.89$; $\mathrm{H}, 7.19$. Found: $\mathrm{C}, 58.91 ; \mathrm{H}, 7.17$.

\section{B2. General procedure for the preparation of 2-substitued 3-} methoxybenzoic acids (10, 12, 16a-d, 21 and 23)

To a stirred solution of LTMP (5 equiv.) in THF (20 mL) at $0{ }^{\circ} \mathrm{C}$ was added dropwise 3-methoxybenzoic acid (6) (0.46 g, $3 \mathrm{mmol})$ in THF (5 mL). After $2 \mathrm{~h}$ stirring at this temperature, the solution was quenched with the electrophile (6-10 equiv). Stirring was maintained for $30 \mathrm{~min}$ and the solution was then allowed to warm to ambient temperature or heated to $40-65{ }^{\circ} \mathrm{C}$ for $2 \mathrm{~h}$. After hydrolysis with water $(30 \mathrm{~mL})$, the aqueous phase was washed with diethyl ether $(20 \mathrm{~mL})$, acidified with aqueous $(2 \mathrm{M}) \mathrm{HCl}$, and extracted with diethyl ether. The organic layer phase dried over $\mathrm{MgSO}_{4}$, filtrated and concentrated in vacuo to give the crude benzoic acids (10, 12, 16a-d, 21 and 23) which were chromatographed on silicagel or recrystallized.

\section{2-Deuterio-3-methoxybenzoic acid (10)}

See general procedure. The solution was quenched with deuterium oxide (0.33 mL, $18 \mathrm{mmol})$. Standard workup afforded 10 (92\% crude yield). ${ }^{1} \mathrm{H}$ NMR $\left(400 \mathrm{MHz}, \mathrm{CDCl}_{3}\right) \rightarrow 3.87(\mathrm{~s}, 3 \mathrm{H}), 7.14(\mathrm{~d}, 1 \mathrm{H}, J=8.4 \mathrm{~Hz}), 7.41(\mathrm{dd}, 1 \mathrm{H}, J=8.4 \mathrm{~Hz}$ and $J=7.8 \mathrm{~Hz}), 7.72(\mathrm{~d}, 1 \mathrm{H}, J=7.8 \mathrm{~Hz})$.

\section{3-Methoxy-2-methylbenzoic acid (12)}

See general procedure. The solution was quenched with iodomethane $(1.9 \mathrm{~mL}$, $30 \mathrm{mmol}$ ). After being stirred for $30 \mathrm{~min}$ at $0{ }^{\circ} \mathrm{C}$, the reaction mixture was heated for $2 \mathrm{~h}$ at $40^{\circ} \mathrm{C}$. Standard workup followed by chromatography (cyclohexane/ethyl 


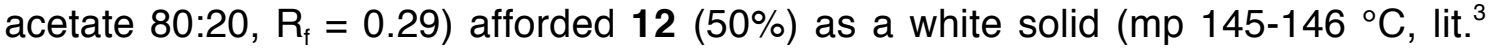
146.5-149.0 $\left.{ }^{\circ} \mathrm{C}\right) .{ }^{1} \mathrm{H}$ NMR $\left(400 \mathrm{MHz}, \mathrm{CDCl}_{3}\right) \rightarrow 2.52(\mathrm{~s}, 3 \mathrm{H}), 3.87(\mathrm{~s}, 3 \mathrm{H}), 7.04(\mathrm{~d}, 1 \mathrm{H}$, $J=8.4 \mathrm{~Hz}$ ), 7.20-7.26 (dd, $1 \mathrm{H}, J=8.4 \mathrm{~Hz}$ and $J=7.8 \mathrm{~Hz}), 7.61(\mathrm{~d}, 1 \mathrm{H}, J=7.8 \mathrm{~Hz})$. ${ }^{13} \mathrm{C}$ NMR $\left(50 \mathrm{MHz}, \mathrm{CDCl}_{3}\right) \rightarrow 13.3,56.3,114.7,123.3,126.5,130.2,130.6,158.5$, 174.3. IR (neat): 2646, $1682,1583 \mathrm{~cm}^{-1}$. Anal. calcd. for $\mathrm{C}_{9} \mathrm{H}_{10} \mathrm{O}_{3}: \mathrm{C}, 65.05 ; \mathrm{H}, 6.07$. Found: $\mathrm{C}, 65.01 ; \mathrm{H}, 6.01$.

\section{2-Chloro-3-methoxybenzoic acid (16a)}

See general procedure. The solution was quenched with hexachloroethane $(4.27 \mathrm{~g}, 18 \mathrm{mmol})$. After being stirred for $30 \mathrm{~min}$ at $0^{\circ} \mathrm{C}$, the mixture was heated at $65^{\circ} \mathrm{C}$ for $2 \mathrm{~h}$. Standard workup followed by chromatography (cyclohexane/ethyl acetate 80:20, $\left.\mathrm{R}_{\mathrm{f}}=0.13\right)$ gave $16 \mathrm{a}(47 \%)$ as a white solid (mp 160-161 $\left.{ }^{\circ} \mathrm{C}\right) .{ }^{1} \mathrm{H}$ NMR $\left(400 \mathrm{MHz}, \mathrm{CDCl}_{3}\right) \rightarrow 3.95(\mathrm{~s}, 3 \mathrm{H}), 7.13(\mathrm{dd}, 1 \mathrm{H}, J=8.4 \mathrm{~Hz}$ and $J=1.5 \mathrm{~Hz}$, 7.32 (dd, $1 \mathrm{H}, J=7.9 \mathrm{~Hz}$ and $J=8.4 \mathrm{~Hz}), 7.56(\mathrm{dd}, 1 \mathrm{H}, J=7.9 \mathrm{~Hz}$ and $J=1.5 \mathrm{~Hz}) \cdot{ }^{13} \mathrm{C}$ NMR $(50$ $\left.\mathrm{MHz}, \mathrm{DMSO}-d_{6}\right) \rightarrow \mid 56.4,114.6,119.3,121.2,127.7,133.8,150.0,167.1$. IR (neat): 2938, 1679, $1574 \mathrm{~cm}^{-1}$. HRMS (EI) $\mathrm{m} / z$ calcd. for $\mathrm{C}_{8} \mathrm{H}_{7} \mathrm{O}_{3} \mathrm{Cl}\left(\mathrm{M}^{+}\right.$.): 186.0083. Found: 186.0076 .

\section{2-Bromo-3-methoxybenzoic acid (16b)}

See general procedure. The solution was quenched with dibromotetrachloroethane $(5.87 \mathrm{~g} 18 \mathrm{mmol})$. After being stirred for $30 \mathrm{~min}$ at $0{ }^{\circ} \mathrm{C}$, the solution was heated at $65{ }^{\circ} \mathrm{C}$ for $2 \mathrm{~h}$. Standard workup followed by chromatography (cyclohexane/ethyl acetate $80: 20, \mathrm{R}_{\mathrm{f}}=0.15$ ) afforded $16 \mathrm{~b}(60 \%)$ as a brown solid (mp 154-155 ${ }^{\circ} \mathrm{C}$, lit $\left.{ }^{4} .155-156{ }^{\circ} \mathrm{C}\right) .{ }^{1} \mathrm{H}$ NMR $\left(400 \mathrm{MHz} \mathrm{CDCl}_{3}\right) \rightarrow 3.98(\mathrm{~s}, 3 \mathrm{H}), 7.07$ (dd, $1 \mathrm{H}, J=7.8 \mathrm{~Hz}$ and $J=1.5 \mathrm{~Hz}$ ), $7.38(\mathrm{t}, 1 \mathrm{H}, J=7.8 \mathrm{~Hz}), 7.52(\mathrm{dd}, 1 \mathrm{H}, J=7.8 \mathrm{~Hz}$ and $J=1.5 \mathrm{~Hz}) .{ }^{13} \mathrm{C}$ NMR $\left(50 \mathrm{MHz}\right.$, DMSO- $\left.d_{6}\right) \rightarrow 56.7,109.1,114.4,121.4,129.0$, 137.0, 156.1, 168.3. IR (neat): $2941,1680,1570 \mathrm{~cm}^{-1}$. Anal. calcd. for $\mathrm{C}_{8} \mathrm{H}_{7} \mathrm{BrO}_{3}: \mathrm{C}$, 
41.59; $\mathrm{H}$, 3.05. Found: $\mathrm{C}, 41.56 ; \mathrm{H}, 3.11$. HRMS (El) $\mathrm{m} / \mathrm{z}$ calcd. for $\mathrm{C}_{8} \mathrm{H}_{7} \mathrm{O}_{3}{ }^{79} \mathrm{Br}\left(\mathrm{M}^{+}\right.$.): 229.9578. Found: 229.9577.

\section{2-lodo-3-methoxybenzoic acid (16c)}

See general procedure. The solution was quenched with iodine $(4.57 \mathrm{~g}$, $18 \mathrm{mmol}$ ). After being stirred for $30 \mathrm{~min}$ at $0{ }^{\circ} \mathrm{C}$, the mixture was heated at $65^{\circ} \mathrm{C}$ for $2 \mathrm{~h}$. Standard workup followed by chromatography (cyclohexane/ethyl acetate 80:20) gave 16c (53\%) as a yellow solid (mp 145-146 ${ }^{\circ} \mathrm{C}$, lit. $\left.{ }^{5} 148-149{ }^{\circ} \mathrm{C}\right) .{ }^{1} \mathrm{H}$ NMR $\left(400 \mathrm{MHz}, \mathrm{CDCl}_{3}\right) \rightarrow 3.94(\mathrm{~s}, 3 \mathrm{H}), 6.99(\mathrm{dd}, 1 \mathrm{H}, J=7.9 \mathrm{~Hz}$ and $J=1.5 \mathrm{~Hz}), 7.38(\mathrm{t}$, $1 \mathrm{H}, J=7.9 \mathrm{~Hz}, 7.49(\mathrm{dd}, 1 \mathrm{H}, J=7.9 \mathrm{~Hz}$ and $J=1.5 \mathrm{~Hz}) .{ }^{13} \mathrm{C}$ NMR $\left(50 \mathrm{MHz}, \mathrm{CDCl}_{3}\right)$ $\rightarrow 56.9,87.5,114.1,123.6,129.3,136.7,158.9,172.5$. IR (neat): 2927, 1738, 1609 $\mathrm{cm}^{-1}$. HRMS (EI) $\mathrm{m} / z$ calcd.for $\mathrm{C}_{8} \mathrm{H}_{7} \mathrm{O}_{3} \mathrm{I}\left(\mathrm{M}^{+}\right.$.): 227.9440 . Found: 277.9445 .

\section{3-Methoxy-2-methylthiobenzoic acid (16d)}

See general procedure. The solution was quenched with dimethyldisulfide (1.6 mL, $18 \mathrm{mmol})$. Standard workup followed by chromatography (cyclohexane/ethyl acetate 60:40, $\left.R_{f}=0.39\right)$ afforded $16 d(46 \%)$ as a brown-red solid (mp 132-134 ${ }^{\circ} \mathrm{C}$ ). ${ }^{1} \mathrm{H}$ NMR (400 MHz, $\left.\mathrm{CDCl}_{3}\right) \rightarrow 2.44(\mathrm{~s}, 3 \mathrm{H}), 3.96(\mathrm{~s}, 3 \mathrm{H}), 7.09$ (dd, $1 \mathrm{H}, J=8.4 \mathrm{~Hz}$ ), $7.40(\mathrm{dd}, 1 \mathrm{H}, J=8.4 \mathrm{~Hz}$ and $J=7.9 \mathrm{~Hz}), 7.65(\mathrm{dd}, 1 \mathrm{H}, J=7.9 \mathrm{~Hz}$ and $J=1.0 \mathrm{~Hz})$. ${ }^{13} \mathrm{C}$ NMR $\left(50 \mathrm{MHz}, \mathrm{CDCl}_{3}\right) \rightarrow 19.2,56.7,114.7,122.9,123.9,129.8,136.3,160.5$, 171.8. IR (neat): 2926, 1739, $1609 \mathrm{~cm}^{-1}$. Anal. calcd. for $\mathrm{C}_{9} \mathrm{H}_{10} \mathrm{O}_{3} \mathrm{~S}: \mathrm{C}, 54.53 ; \mathrm{H}, 5.08$. Found: $\mathrm{C}, 54.28 ; \mathrm{H}, 5.13$.

\section{3-Hydroxy-4-methoxyisobenzofuran-1(3H)-one (21)}

See general procedure. The solution was quenched with $N, N$ dimethylformamide (18 mmol, $1.4 \mathrm{~mL}$ ). Usual workup followed by chromatography (cyclohexane/ethyl acetate 60:40, $\left.\mathrm{R}_{\mathrm{f}}=0.24\right)$ afforded $21(27 \%)$ as a white solid (mp 154-155 ${ }^{\circ} \mathrm{C}$, lit. $\left.{ }^{7} 156-157{ }^{\circ} \mathrm{C}\right) .{ }^{1} \mathrm{H}$ NMR (400 MHz, $\left.\mathrm{CDCl}_{3}\right) \rightarrow 3,96$ (s, 3H), 6.70 (s,

5 Stanley, W. M.; McMahon, E.; Roger, A. J. Am. Chem. Soc. 1933, 55, 706. 
1H), $7.17(\mathrm{~d}, 1 \mathrm{H}, J=7.9 \mathrm{~Hz}), 7.47(\mathrm{~d}, 1 \mathrm{H}, J=6.9 \mathrm{~Hz}), 7.57$ (dd, $1 \mathrm{H}, J=7.9 \mathrm{~Hz}, J=$ $6.9 \mathrm{~Hz}) .{ }^{13} \mathrm{C}$ NMR $\left(50 \mathrm{MHz}, \mathrm{DMSO}-d_{6}\right) \rightarrow 56.2,97.5,116.4,117.3,128.7,133.0$, $134.5,155.5,168,8$.

\section{4-Methoxy-3-phenylisobenzofuran-1(3H)-one (22)}

See general procedure. The solution was quenched with benzaldehyde (1.84 mL, $18 \mathrm{mmol})$. Standard workup followed by chromatography (cyclohexane/

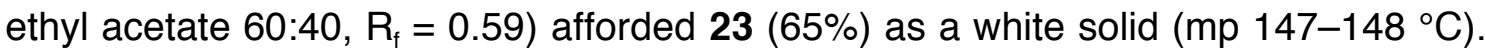
${ }^{1} \mathrm{H}$ NMR $\left(400 \mathrm{MHz}, \mathrm{CDCl}_{3}\right) \rightarrow 3.74(\mathrm{~s}, 3 \mathrm{H}), 6.40$ (s, 1H), 7.08 (dd, $1 \mathrm{H}, J=7.5 \mathrm{~Hz}$ and $J=1.5 \mathrm{~Hz}), 7.25-7.28(\mathrm{~m}, 1 \mathrm{H}), 7.33-7.35(\mathrm{~m}, 1 \mathrm{H}), 7.5-7.56(\mathrm{~m}, 2 \mathrm{H}) .{ }^{13} \mathrm{C}$ NMR $\left(100 \mathrm{MHz}, \mathrm{CDCl}_{3}\right) \rightarrow 55.6,81.7,115.6,117.1,127.1,128.8,129.0,131.4,135.7$, 137.2, 154.6, 170.5. IR (neat): 2926, $1739,1609 \mathrm{~cm}^{-1}$. Anal. calcd. for $\mathrm{C}_{15} \mathrm{H}_{12} \mathrm{O}_{3}: \mathrm{C}$, 74.99; H, 5.03. Found: C, 74.56; H, 5.05.

\section{2,6-Di(trimethylsilyl)-3-methoxybenzoic acid (15) (entry 5 of Table 1)}

To a stirred solution of LTMP $(15 \mathrm{mmol})$ in THF $(20 \mathrm{~mL})$ at $0{ }^{\circ} \mathrm{C}$ was added successively $6(0.46 \mathrm{~g}, 3 \mathrm{mmol})$ in THF $(5 \mathrm{~mL})$ and chlorotrimethylsilane $(1.38 \mathrm{~mL}$, $10.5 \mathrm{mmol})$. After being stirred for $2 \mathrm{~h}$ at $0^{\circ} \mathrm{C}$, the solution was allowed to warm to ambient temperature. Aqueous (2M) $\mathrm{NaOH}$ was added up to $\mathrm{pH} 10$, the aqueous layer was washed with diethyl ether, acidified with aqueous (4M) $\mathrm{HCl}$, and extracted with diethyl ether. The organic layer was dried over $\mathrm{MgSO}_{4}$, filtrated, and concentrated in vacuo. The crude acid 15 was purified by chromatography (cyclohexane/ethyl acetate $\left.90: 10, R_{f}=0.29\right)$ to give a yellow solid $(51 \%)(\mathrm{mp}$ 142-144 $\left.{ }^{\circ} \mathrm{C}\right)$. Proof for the regiochemistry of 15 was gathered by qualitative homonuclear NOE difference spectroscopy. Thus, individual irradiations of $\mathrm{H}-4$ showed a large enhancement of the neighboring methoxy group (10.5\%). ${ }^{1} \mathrm{H}$ NMR $\left(400 \mathrm{MHz}, \mathrm{CDCl}_{3}\right) \rightarrow 0.31(\mathrm{~s}, 9 \mathrm{H}), 0.33(\mathrm{~s}, 9 \mathrm{H}), 3.82(\mathrm{~s}, 3 \mathrm{H}), 6.91(\mathrm{~d}, 1 \mathrm{H}, J=8.4 \mathrm{~Hz})$, $7.58(\mathrm{~d}, 1 \mathrm{H}, J=8.4 \mathrm{~Hz}) .{ }^{13} \mathrm{C}$ NMR $\left(100 \mathrm{MHz} \mathrm{CDCl}_{3}\right) \rightarrow 0.5,1.3,55.2,111.0,126.1$, 128.8, 137.7, 145.1, 165.3, 178.0. IR (neat): $2940,1679 \mathrm{~cm}^{-1}$. 


\section{General procedure for the preparation of 4-substituted 3-methoxybenzoic acids (13 and 17a-d)}

3-Methoxybenzoic acid ( $0.46 \mathrm{~g}, 3 \mathrm{mmol})$ in THF $(5 \mathrm{~mL})$ was added dropwise to a stirred solution of $n$-BuLi/t-BuOK (1:1 ratio, 12 equiv) in THF (30 mL) at $-78{ }^{\circ} \mathrm{C}$. After being stirred for $2 \mathrm{~h}\left(-78^{\circ} \mathrm{C} \rightarrow-50^{\circ} \mathrm{C}\right)$, the reaction mixture was quenched with the electrophile $(18 \mathrm{mmol})$. Stirring was maintained for $30 \mathrm{~min}$ at $-50{ }^{\circ} \mathrm{C}$, the resulting mixture was allowed to warm to rt and hydrolyzed with water $(30 \mathrm{~mL})$. The aqueous layer was washed with diethyl ether $(20 \mathrm{~mL})$, acidified with aqueous $(2 \mathrm{M}) \mathrm{HCl}$, and extracted with diethyl ether. The organic layer was dried over $\mathrm{MgSO}_{4}$, filtrated and concentrated in vacuo to give the crude benzoic acids (13 and 17a-d) which were purified by chromatography or fractional crystallization.

\section{3-Methoxy-4-methylbenzoic acid (13)}

See general procedure. The solution was quenched with iodomethane $(1.12 \mathrm{~mL}, 18 \mathrm{mmol})$. Standard workup followed by fractional crystallization (cyclohexane/ethyl acetate) led to $13(59 \%)$ as a white solid ( $\mathrm{mp} 210-213^{\circ} \mathrm{C}$, lit. ${ }^{6}$ 212-214 $\left.{ }^{\circ} \mathrm{C}\right) .{ }^{1} \mathrm{H}$ NMR $\left(200 \mathrm{MHz}, \mathrm{CDCl}_{3}\right) \rightarrow 2.27(\mathrm{~s}, 3 \mathrm{H}), 3.89(\mathrm{~s}, 3 \mathrm{H}), 7.18(\mathrm{~d}, 1 \mathrm{H}, J=$ $7.8 \mathrm{~Hz}), 7.58(\mathrm{~s}, 1 \mathrm{H}), 7.74(\mathrm{~d}, 1 \mathrm{H}, J=7.8 \mathrm{~Hz}) .{ }^{13} \mathrm{C}$ NMR $\left(100 \mathrm{MHz}\right.$, DMSO- $\left.d_{6}\right) \rightarrow 16.5$, 55.6, 110.7, 121.9, 130.0, 130.1, 130.7, 157.4, 167.6. IR (neat): 2960, 1673, $1609 \mathrm{~cm}^{-1}$. HRMS (El) $\mathrm{m} / z$ calcd. for $\mathrm{C}_{9} \mathrm{H}_{10} \mathrm{O}_{3}\left(\mathrm{M}^{+}\right.$.): 166.0630. Found: 166.0631 .

\section{4-Chloro-3-methoxybenzoic acid (17a)}

See general procedure. The solution was quenched with hexachloroethane $(4.26 \mathrm{~g}, 18 \mathrm{mmol})$. Standard workup followed by fractional crystallization (cyclohexane/ethyl acetate) led to $17 \mathrm{a}(39 \%)$ as a brown solid $\left(\mathrm{mp} 210-212{ }^{\circ} \mathrm{C}\right) .{ }^{1} \mathrm{H}$ $\operatorname{NMR}\left(200 \mathrm{MHz}, \mathrm{CDCl}_{3}\right) \rightarrow 3.98(\mathrm{~s}, 3 \mathrm{H}), 7.48(\mathrm{~d}, 1 \mathrm{H}, J=7.8 \mathrm{~Hz}), 7.62(\mathrm{~s}, 1 \mathrm{H}), 7.69$ (dd, $1 \mathrm{H}, J=7.8 \mathrm{~Hz}$ and $J=1.5 \mathrm{~Hz}) .{ }^{13} \mathrm{C}$ NMR $\left(50 \mathrm{MHz}\right.$, DMSO- $\left.d_{6}\right) \rightarrow 56.1,112.7$, 122.3, 125.9, 129.9, 130.9, 154.4, 166.5. IR (neat): $2975,1678 \mathrm{~cm}^{-1}$.

\footnotetext{
${ }^{6}$ Bennetau, B.; Mortier, J.; Moyroud, J.; Guesnet, J.-L. J. Chem. Soc. Perkin Trans. 1 1995, 1265.
} 


\section{4-Bromo-3-methoxybenzoic acid (17b)}

See general procedure. The solution was quenched with dibromotetrachloroethane $(5.87 \mathrm{~g}, 18 \mathrm{mmol}$,$) . Standard workup followed by fractional$ crystallization (cyclohexane/ethyl acetate) afforded 17b (65\%) as a white solid (mp 212-213이 ${ }^{\circ}{ }^{1} \mathrm{H}$ NMR $\left(400 \mathrm{MHz}, \mathrm{CDCl}_{3}\right) \rightarrow 3.99(\mathrm{~s}, 3 \mathrm{H}), 7.64(\mathrm{~s}, 1 \mathrm{H}), 7.67(\mathrm{~m}, 1 \mathrm{H})$ ${ }^{13} \mathrm{C}$ NMR $\left(100 \mathrm{MHz}\right.$, DMSO- $\left.d_{6}\right) \rightarrow 56.1,112.5,116.0,122.3,130.9,132.1,155.4$, 166.6. IR (neat): $2970,1679 \mathrm{~cm}^{-1}$.

\section{4-lodo-3-methoxybenzoic acid (17c)}

See general procedure. The solution was quenched with iodine $(4.57 \mathrm{~g}$, $18 \mathrm{mmol}$ ). Standard workup followed by fractional crystallization (cyclohexane/ethyl acetate) afforded $17 \mathrm{c}(20 \%)$ as a white solid (mp 210-212 $\left.{ }^{\circ} \mathrm{C}\right) .{ }^{1} \mathrm{H}$ NMR $(400 \mathrm{MHz}$, $\left.\mathrm{CDCl}_{3}\right) \rightarrow 4.04(\mathrm{~s}, 3 \mathrm{H}), 7.45(\mathrm{dd}, 1 \mathrm{H}, J=8.4 \mathrm{~Hz}$ and $J=1.5 \mathrm{~Hz}), 7.57(\mathrm{~d}, 1 \mathrm{H}, J=1.5$ $\mathrm{Hz}), 8.06(\mathrm{~d}, 1 \mathrm{H}, J=8.4 \mathrm{~Hz}) .{ }^{13} \mathrm{C}$ NMR $\left(50 \mathrm{MHz}, \mathrm{DMSO}-d_{6}\right) \rightarrow 56.4,92.6,111.2$, 123.2, 132.5, 139.3, 157.9, 166.8. IR (neat): $2960,1673,1608 \mathrm{~cm}^{-1}$.

\section{3-Methoxy-4-thiomethylbenzoic acid (17d)}

See general procedure. The solution was quenched with dimethyldisulfide (18 mmol, $1.6 \mathrm{~mL})$. Standard workup followed by fractional crystallization (cyclohexane/ethyl acetate) afforded $17 d(51 \%)$ as a yellow solid (mp $186-187^{\circ} \mathrm{C}$ ). ${ }^{1} \mathrm{H}$ NMR (400 MHz, $\mathrm{CDCl}_{3}$ ) $\rightarrow 2.48(\mathrm{~s}, 3 \mathrm{H}), 3.97$ (s, 3H), $7.16(\mathrm{~d}, 1 \mathrm{H}, J=8.4 \mathrm{~Hz}), 7.51$ $(\mathrm{d}, 1 \mathrm{H}, J=1.5 \mathrm{~Hz}), 7.75(\mathrm{dd}, 1 \mathrm{H}, J=8.4 \mathrm{~Hz}$ and $J=1.5 \mathrm{~Hz}) .{ }^{13} \mathrm{C}$ NMR $(50 \mathrm{MHz}$, $\left.\mathrm{CDCl}_{3}\right) \rightarrow 19.2,56.7,114.7,122.9,123.9,129.8,136.3,160.5,171.8$. IR (neat): 2970, 1674, $1592 \mathrm{~cm}^{-1}$. HRMS (EI) $\mathrm{m} / \mathrm{z}$ calcd. for $\mathrm{C}_{9} \mathrm{H}_{10} \mathrm{O}_{3} \mathrm{~S}\left(\mathrm{M}^{+}.\right)$: 198.0351. Found: 198.0361.

\section{4-(Hydroxy(phenyl)methyl)-3-methoxybenzoic acid (17f)}

See general procedure. The solution was quenched with benzaldehyde (18 mmol, $1.84 \mathrm{~mL}$ ). Standard workup followed by chromatography (cyclohexane/ 
ethyl acetate 60:40, $\left.R_{f}=0.28\right)$ gave $17 f(54 \%)$ as a yellow solid (mp 122-124 ${ }^{\circ} \mathrm{C}$ ). ${ }^{1} \mathrm{H}$ NMR $\left(400 \mathrm{MHz} \mathrm{CDCl}_{3}\right) \rightarrow 3.87(\mathrm{~s}, 3 \mathrm{H}), 6.11(\mathrm{~s}, 1 \mathrm{H}), 7.27-7.42(\mathrm{~m}, 5 \mathrm{H}), 7.47$ (d, $1 \mathrm{H}, J=7.8 \mathrm{~Hz}) .7 .58(\mathrm{~d}, 1 \mathrm{H}, J=1.6 \mathrm{~Hz}), 7.75(\mathrm{dd}, 1 \mathrm{H}, J=7.8 \mathrm{~Hz}$ and $J=1.6 \mathrm{~Hz})$. ${ }^{13} \mathrm{C}$ NMR $\left(100 \mathrm{MHz}\right.$, DMSO- $\left.d_{6}\right) \rightarrow 26.3,55.5,67.9,110.9,121.9,126.4,126.7,128.0$, 130.3, 138.6, 144.6, 155.3, 167.1. IR (neat): 3342, 2927, $1677 \mathrm{~cm}^{-1}$.

\section{General procedure for the preparation of 6-substituted-3-methoxy-2- (trimethylsilyl)benzoic acids (18, 19a-e and 22) and 6-substitued 3- methoxybenzoic acids (14 and 20a-d)}

To a stirred solution of $s$-BuLi/TMEDA $(4 \mathrm{mmol})$ in dry THF $(5 \mathrm{~mL})$ at $-78{ }^{\circ} \mathrm{C}$ was added dropwise 3-methoxy-2-(trimethylsilyl)benzoic acid (11) (0.224 g, $1 \mathrm{mmol})$ in dry THF $(5 \mathrm{~mL})$. After being stirred for $2 \mathrm{~h}\left(-78^{\circ} \mathrm{C} \rightarrow-30^{\circ} \mathrm{C}\right)$, the reaction mixture was quenched with the electrophile $(6 \mathrm{mmol})$. Stirring was maintained for $30 \mathrm{~min}$ at $-30^{\circ} \mathrm{C}$, the resulting mixture was allowed to warm to $\mathrm{rt}$ and hydrolyzed with water $(20 \mathrm{~mL})$. The aqueous layer was washed with diethyl ether $(20 \mathrm{~mL})$, acidified with aqueous $(2 \mathrm{M}) \mathrm{HCl}$ until the $\mathrm{pH}$ reached 2, and extracted with diethyl ether. The organic layer was dried over $\mathrm{MgSO}_{4}$, filtrated and concentrated in vacuo to give the crude benzoic acids (18, 19a-e and 22) which were purified by column chromatography or recrystallization. The acids 14 and 20 a-d were prepared in a similar manner. The crude reaction mixture was hydrolyzed with aqueous $(6 \mathrm{M}) \mathrm{HCl}$ to $\mathrm{pH} 1$.

\section{6-Methyl-3-methoxy-2-(trimethylsilyl)benzoic acid (18)}

See general procedure. The solution was quenched with iodomethane $(0.38 \mathrm{~mL}, 6 \mathrm{mmol})$. Standard workup followed by recrystallization (cyclohexane/ethyl acetate) afforded $18(57 \%)$ as a white solid (mp 124.5-125.5 $\left.{ }^{\circ} \mathrm{C}\right) .{ }^{1} \mathrm{H}$ NMR $(400 \mathrm{MHz}$, $\left.\mathrm{CDCl}_{3}\right) \rightarrow 0.33(\mathrm{~s}, 9 \mathrm{H}), 3.82(\mathrm{~s}, 3 \mathrm{H}), 6.82(\mathrm{~d}, 1 \mathrm{H}, J=8.4 \mathrm{~Hz}), 7.23(\mathrm{~d}, 1 \mathrm{H}, J=8,4 \mathrm{~Hz})$. ${ }^{13} \mathrm{C}$ NMR $\left(50 \mathrm{MHz}, \mathrm{CDCl}_{3}\right) \rightarrow 0.7,18.2,54.8,110.8,123.9,125.3,131.9,138.8$, 
162.1, 176.8. IR (neat): 2939, 2571, $1695 \mathrm{~cm}^{-1}$. Anal. calcd. for $\mathrm{C}_{12} \mathrm{H}_{18} \mathrm{SiO}_{3}: \mathrm{C}, 60.47$; $H, 7.61$. Found: $C, 60.47 ; H, 7.77$.

\section{6-Chloro-3-methoxy-2-(trimethylsilyl)benzoic acid (19a)}

See general procedure. The solution was quenched with hexachloroethane $(1.42 \mathrm{~g}, 6 \mathrm{mmol})$. Standard workup followed by chromatography (cyclohexane/ethyl acetate 90:10, $\left.R_{f}=0.35\right)$ afforded $19 a(57 \%)$ as a colorless solid $\left(\mathrm{mp} 151.5-153^{\circ} \mathrm{C}\right)$. ${ }^{1} \mathrm{H}$ NMR (200 MHz, $\left.\mathrm{CDCl}_{3}\right) \rightarrow 0.33(\mathrm{~s}, 9 \mathrm{H}), 3.81(\mathrm{~s}, 3 \mathrm{H}), 6.83(\mathrm{~d}, 1 \mathrm{H}, J=8.8 \mathrm{~Hz}), 7.38$ $(\mathrm{d}, 1 \mathrm{H}, J=8.8 \mathrm{~Hz}) .{ }^{13} \mathrm{C} \mathrm{NMR}\left(50 \mathrm{MHz}, \mathrm{CDCl}_{3}\right) \rightarrow 1.65,55.4,112.0,121.6,126.8$, 131.0, 138.5, 162.8, 173.8. IR (neat): 2976, $1693 \mathrm{~cm}^{-1}$. Anal. calcd. for $\mathrm{C}_{11} \mathrm{H}_{15} \mathrm{ClO}_{3} \mathrm{Si}$ : C, 51.06; H, 5.84. Found: C, 50.64; H, 5.89 .

\section{6-Bromo-3-methoxy-2-(trimethylsilyl)benzoic acid (19b)}

See general procedure. The solution was quenched with dibromotetrachloroethane $(1.96 \mathrm{~g}, 6 \mathrm{mmol})$. Standard workup followed by recrystallization ( $n$ heptane/ethyl acetate) afforded $19 \mathrm{~b}(66 \%)$ as a colorless solid (mp $\left.162-164{ }^{\circ} \mathrm{C}\right) .{ }^{1} \mathrm{H}$ $\operatorname{NMR}\left(200 \mathrm{MHz}, \mathrm{CDCl}_{3}\right) \rightarrow 0.33(\mathrm{~s}, 9 \mathrm{H}), 3.81(\mathrm{~s}, 3 \mathrm{H}), 6.78(\mathrm{~d}, 1 \mathrm{H}, J=8.8 \mathrm{~Hz}), 7.53(\mathrm{~d}$, $1 \mathrm{H}, J=8.8 \mathrm{~Hz}) .{ }^{13} \mathrm{C} \mathrm{NMR}\left(50 \mathrm{MHz}, \mathrm{CDCl}_{3}\right) \rightarrow 0.54,55.3,109.5,112.4,127.1,134.2$, 140.3, 163.2, 174.7. IR (neat): 2980, $1694 \mathrm{~cm}^{-1}$. Anal. calcd. for $\mathrm{C}_{11} \mathrm{H}_{15} \mathrm{IO}_{3} \mathrm{Si}$ : C, 43.57; H, 4.99. Found: C, 43.98; H, 5.11.

\section{6-lodo-3-methoxy-2-(trimethylsilyl)benzoic acid (19c)}

See general procedure. The solution was quenched with iodine $(1.52 \mathrm{~g}$, $6 \mathrm{mmol}$ ). Standard workup followed by chromatography (cyclohexane/ethyl acetate 90:10, $\left.R_{f}=0.25\right)$ afforded $19 \mathrm{c}(53 \%)$ as a white solid (mp 152-154 $\left.{ }^{\circ} \mathrm{C}\right) .{ }^{1} \mathrm{H}$ NMR $\left(200 \mathrm{MHz}, \mathrm{CDCl}_{3}\right) \rightarrow 0.32(\mathrm{~s}, 9 \mathrm{H}), 3.80(\mathrm{~s}, 3 \mathrm{H}), 6.64(\mathrm{~d}, 1 \mathrm{H}, J=8.8 \mathrm{~Hz}), 7.79(\mathrm{~d}, 1 \mathrm{H}, J$ $=8.8 \mathrm{~Hz}) .{ }^{13} \mathrm{C} \mathrm{NMR}\left(50 \mathrm{MHz}, \mathrm{CDCl}_{3}\right) \rightarrow 1.01,55.1,81.1,112.7,121.2,140.8,144.4$, 164.0, 175.6. IR (neat): 2966, 2570, $1687 \mathrm{~cm}^{-1}$. Anal. calcd. for $\mathrm{C}_{11} \mathrm{H}_{15} \mathrm{IO}_{3} \mathrm{Si}$ : C, 37.72 ; $\mathrm{H}$, 4.32. Found: $\mathrm{C}, 37.91 ; \mathrm{H}, 4.24$. 


\section{3-Methoxy-6-thiomethyl-2-(trimethylsilyl)benzoic acid (19d)}

See general procedure. The solution was quenched with dimethyldisulfide (6 mmol, $0.53 \mathrm{~mL}$ ). Standard workup followed by recrystallization (cyclohexane/ethyl acetate) afforded 19d (61\%) as a brown solid (mp 152-154 $\left.{ }^{\circ} \mathrm{C}\right) .{ }^{1} \mathrm{H}$ NMR $(200 \mathrm{MHz}$, $\left.\mathrm{CDCl}_{3}\right) \rightarrow 0.33(\mathrm{~s}, 9 \mathrm{H}), 2.41(\mathrm{~s}, 3 \mathrm{H}), 3.81(\mathrm{~s}, 3 \mathrm{H}), 6.87(\mathrm{~d}, 1 \mathrm{H}, J=8.7 \mathrm{~Hz}), 7.52(\mathrm{~d}$, $1 \mathrm{H}, J=8.7 \mathrm{~Hz}) .{ }^{13} \mathrm{C}$ NMR $\left(50 \mathrm{MHz}, \mathrm{CDCl}_{3}\right) \rightarrow 0.87,20.2,54.9,110.7,123.7,124.6$, 135.2, 145.0, 163.6, 174.2. IR (neat): 2924, 2634, $1695 \mathrm{~cm}^{-1}$. HRMS (EI) $\mathrm{m} / \mathrm{z}$ calcd. for $\mathrm{C}_{12} \mathrm{H}_{18} \mathrm{O}_{3} \mathrm{SiS}\left(\mathrm{M}^{+}.\right)$: 270.0746. Found: 270.0741 .

\section{3-Hydroxy-6-methoxy-7-(trimethylsilyl) isobenzofuran-1(3H)-one (23)}

See general procedure. The solution was quenched with $N, N$ dimethylformamide $(0.37 \mathrm{~mL}, 6 \mathrm{mmol})$. Standard workup followed by chromatography (cyclohexane/ethyl acetate 60:40, $\mathrm{R}_{\mathrm{f}}=0.34$ ) afforded $23(20 \%)$ as a colorless solid. ${ }^{1} \mathrm{H}$ NMR (200 MHz, $\mathrm{CDCl}_{3}$ ) $\rightarrow 0.39$ (s, 9H), 3.82 (s, 3H), 7.09 (d, 1H, J=8.3 Hz), 7.58 $\left(\mathrm{d}, 1 \mathrm{H}, J=8.3 \mathrm{~Hz}\right.$ ). HRMS (El) $\mathrm{m} / z$ calcd. for $\mathrm{C}_{11} \mathrm{H}_{13} \mathrm{O}_{4} \mathrm{Cl}\left(\mathrm{M}^{+}\right.$.) 237.0583. Found: 237.0591.

\section{6-Methyl-3-methoxybenzoic acid (14)}

See general procedure. After purification by recrystallization ( $n$-heptane/ethyl acetate), the acid 14 was obtained as a white solid (54\%) (mp 151-152 ${ }^{\circ} \mathrm{C}$, lit. ${ }^{7} 151$ $\left.151.5^{\circ} \mathrm{C}\right) .{ }^{1} \mathrm{H}$ NMR $\left(200 \mathrm{MHz}, \mathrm{CDCl}_{3}\right) \rightarrow 2.59(\mathrm{~s}, 3 \mathrm{H}), 3.83(\mathrm{~s}, 3 \mathrm{H}), 7.02(\mathrm{dd}, 1 \mathrm{H}, \mathrm{J}=$ $8.4 \mathrm{~Hz}$ and $J=2.9 \mathrm{~Hz}), 7.16(\mathrm{~d}, 1 \mathrm{H}, J=8.4 \mathrm{~Hz}), 7.58(\mathrm{~d}, 1 \mathrm{H}, J=2.9 \mathrm{~Hz}) .{ }^{13} \mathrm{C} \mathrm{NMR}$ $\left(50 \mathrm{MHz}, \mathrm{CDCl}_{3}\right) \rightarrow 20.6,55.6,115.2,117.9,121.9,131.0,132.7,157.4,168.9$. HRMS (EI) $m / z$ calcd. for $\mathrm{C}_{9} \mathrm{H}_{10} \mathrm{O}_{3}\left(\mathrm{M}^{+}\right.$.): 166.0630. Found: 166.0628 .

(a) Newman, M. S.; Bayerlein, F. J. Org. Chem. 1963, 28, 2804. (b) Roberts, J. D.; Yancey, J. A. J. Am. Chem. Soc. 1951, 73, 1011. 


\section{6-Chloro-3-methoxybenzoic acid (20a)}

See general procedure. After recrystallization (cyclohexane/ethyl acetate), the acid 20a was obtained as a colorless solid (55\%) (mp 168-169.5 ${ }^{\circ} \mathrm{C}$, lit. $^{8} 169-171$ $\left.{ }^{\circ} \mathrm{C}\right) .{ }^{1} \mathrm{H}$ NMR $\left(200 \mathrm{MHz}, \mathrm{CDCl}_{3}\right) \rightarrow 3.85(\mathrm{~s}, 3 \mathrm{H}), 7.02(\mathrm{dd}, 1 \mathrm{H}, J=8.8 \mathrm{~Hz}$ and $J=3.1$ $\mathrm{Hz}), 7.38(\mathrm{~d}, 1 \mathrm{H}, J=8.8 \mathrm{~Hz}), 7.52(\mathrm{~d}, 1 \mathrm{H}, J=3.1 \mathrm{~Hz}) \cdot{ }^{13} \mathrm{C}$ NMR $\left(50 \mathrm{MHz}, \mathrm{CDCl}_{3}\right) \rightarrow$ 55.6, 115.5, 118.3, 122.6, 131.4, 132.2, 157.7, 166.5. IR (neat): 2945, $1672 \mathrm{~cm}^{-1}$. HRMS (El) $m / z$ calcd. for $\mathrm{C}_{8} \mathrm{H}_{7} \mathrm{O}_{3} \mathrm{Cl}\left(\mathrm{M}^{+}.\right)$: 186.0084. Found: 186.0094 .

\section{6-Bromo-3-methoxybenzoic acid (20b)}

See general procedure. After chromatography (cyclohexane/ethyl acetate 80:20, $\left.R_{f}=0.15\right)$, the acid $20 b$ was obtained as a colorless solid (63\%) ( $\mathrm{mp} \mathrm{158-160}$ ${ }^{\circ} \mathrm{C}$, lit. $\left.{ }^{9} 160{ }^{\circ} \mathrm{C}\right) .{ }^{1} \mathrm{H}$ NMR $\left(200 \mathrm{MHz}, \mathrm{CDCl}_{3}\right) \rightarrow 3.83(\mathrm{~s}, 3 \mathrm{H}), 6.95(\mathrm{dd}, 1 \mathrm{H}, \quad J=8.8 \mathrm{~Hz}$ and $J=3.1 \mathrm{~Hz}), 7.54(\mathrm{~d}, 1 \mathrm{H}, J=3.1 \mathrm{~Hz}), 7.59(\mathrm{~d}, 1 \mathrm{H}, J=8.8 \mathrm{~Hz}) .{ }^{13} \mathrm{C}$ NMR $(50 \mathrm{MHz}$, $\left.\mathrm{CDCl}_{3}\right) \rightarrow 55.7,112.9,117.1,120.6,130.9,135.6,158.6,170.9$.

\section{6-lodo-3-methoxybenzoic acid (20c)}

See general procedure. After chromatography (cyclohexane/ethyl acetate $\left.80: 20, R_{f}=0.16\right)$, the acid 20c was obtained as a white solid $(50 \%)$ (mp 132.5-134 $\left.{ }^{\circ} \mathrm{C}\right) .{ }^{1} \mathrm{H}$ NMR $\left(200 \mathrm{MHz}, \mathrm{CDCl}_{3}\right) \rightarrow 3.84(\mathrm{~s}, 3 \mathrm{H}), 6.81$ (dd, $1 \mathrm{H}, J=8.8 \mathrm{~Hz}$ and $J=3.1 \mathrm{~Hz}), 7.57(\mathrm{~d}, 1 \mathrm{H}, J=3.1 \mathrm{~Hz}), 7.89(\mathrm{~d}, 1 \mathrm{H}, J=8.8 \mathrm{~Hz}) .{ }^{13} \mathrm{C} \mathrm{NMR}(50 \mathrm{MHz}$, $\left.\mathrm{CDCl}_{3}\right) \rightarrow 55.6,83.1,117.2,120.5,133.9,142.5,159.5,171.1$. IR (neat): 2934, 2559, $1690 \mathrm{~cm}^{-1}$. Anal. calcd. for $\mathrm{C}_{8} \mathrm{H}_{7} \mathrm{IO}_{3}: \mathrm{C}, 34.56 ; \mathrm{H}, 2.54$. Found: $\mathrm{C}, 34.37 ; \mathrm{H}, 2.71$.

\section{3-Methoxy-6-thiomethylbenzoic acid (20d)}

See general procedure. After recrystallization ( $n$-heptane/ethyl acetate), the acid $20 \mathrm{~d}$ was obtained was a brown solid (61\%) (mp 144-146 $\left.{ }^{\circ} \mathrm{C}\right) .{ }^{1} \mathrm{H}$ NMR $\left(200 \mathrm{MHz}, \mathrm{CDCl}_{3}\right) \rightarrow 2.48(\mathrm{~s}, 3 \mathrm{H}), 3.89(\mathrm{~s}, 3 \mathrm{H}), 7.09(\mathrm{dd}, 1 \mathrm{H}, J=8.8 \mathrm{~Hz}$ and $J=2.8$ $\mathrm{Hz}), 7.23(\mathrm{~d}, 1 \mathrm{H}, J=8.8 \mathrm{~Hz}), 7.65(\mathrm{~d}, 1 \mathrm{H}, J=2.8 \mathrm{~Hz}) .{ }^{13} \mathrm{C} \mathrm{NMR}\left(50 \mathrm{MHz}, \mathrm{CDCl}_{3}\right) \rightarrow$ 
16.8, 56.0, 116.9, 121.1, 127.3, 127.5, 134.9, 156.9, 171.1. IR (neat): 2970, 2567, $1673 \mathrm{~cm}^{-1}$. HRMS (EI) $\mathrm{m} / \mathrm{z}$ calcd. for $\mathrm{C}_{9} \mathrm{H}_{10} \mathrm{O}_{3} \mathrm{~S}\left(\mathrm{M}^{+}\right.$.): 198.0351. Found: 198.0358 . 